\title{
SEPARATION THEOREMS WITH APPLICATIONS TO QUESTIONS CONCERNING ACCESSIBILITY AND PLANE CONTINUA*
}

\author{
BY
}

R. G. LUBBEN

In this paper we shall confine ourselves to a two-dimensional euclidean space which we shall denote by the symbol $S$.

R. L. Moore $\dagger$ has given the following extension of a theorem by Zoretti: $\ddagger$ If $K$ is a bounded maximal connected subset of a closed point set $M$ and does not separate space, and e is a positive number, then there exists a simple closed curve containing no point of $M$, such that the interior of this curve contains $K$ but contains no point whose distance from $K$ is greater than $e$. Zoretti's conclusion is weaker than Moore's in that it says nothing about the points on the interior of the curve surrounding $K$. On the other hand, Moore finds it necessary to use a stronger hypothesis than Zoretti's; namely that $K$ does not separate the plane. In this paper we consider among other things the question of the conditions involved in a combination of Zoretti's hypothesis and Moore's conclusion, and show in Theorem 1 that a result analogous to

* Various parts of this paper were presented to the American Mathematical Society on September 10, 1925, December 30, 1925, and February 27, 1926; received by the editors August 31, 1928.

$\dagger$ Cf. R. L. Moore, Concerning the separation of point sets by curves, Proceedings of the National Academy of Sciences, vol. 11 (1925), pp. 469-476.

$\ddagger$ Cf. L. Zoretti, Sur les fonctions analytiques uniformes, Journal de Mathématiques Pures et Appliquées, (6), vol. 1 (1905), pp. 9-11. A pair of point sets $M_{1}$ and $M_{2}$ are said to be mutually separated provided that neither contains a point or a limit point of the other. A point set is said to be connected if it is not the sum of two non-vacuous mutually separated point sets. A point set $M$ is said to be strongly connected provided that for every pair of points belonging to $M$ there exists a closed, connected subset of $M$ containing these points; cf. R. L. Moore, Concerning continuous curves in the plane, Mathematische Zeitschrift, vol. 15 (1922), p. 254. If $H$ is a point set and $M$ is a connected subset of $H$ such that every connected subset of $H$ which has a point in common with $M$ is a subset of $M$, then $M$ is said to be a maximal connected subset of $H$. A point set $M$ is said to separate space if $S-M$ is not connected. A simple continuous arc $A X B$, whose end points are the distinct points $A$ and $B$, is a closed, bounded, connected set of points, $M$, containing $A$ and $B$ such that if $P$ is a point of $M-A-B, M-P$ is not connected; 'cf. R. L. Moore, Concerning simple continuous curves, these Transactions, vol. 21 (1920), p. 340. The point set $M-A-B$ mentioned above is the segment $A X B$ of the simple continuous arc $A X B$. For definitions of a simple closed curve, of an open curve, and of other of the simpler point sets see the paper mentioned above, the paper F. A., and articles to which references are made in these two papers. We shall use the notation F. A. to denote R. L. Moore's paper, On the foundations of plane analysis situs, these Transactions, vol. 17 (1916), pp. 131-164. 
that of Moore holds, if we allow the boundary of the domain covering $K$ to consist of a finite number of simple closed curves. In Theorems 2 and 3 we give conditions under which it is possible to cover a bounded point set by a finite number of such domains.

It follows from these theorems that a given point in one of two closed, bounded, mutually exclusive point sets can be separated from a given point in the other by a simple closed curve containing no point of the sum of the two point sets. In problems concerning the boundaries of domains, accessibility, and the separation of unbounded point sets by curves, it is of interest to consider the question of the separation of sets, having points in common, by simple closed curves containing no points of the given sets, except those that are common to them. R. L. Moore* has given sufficient conditions for the existence of such a curve, for the case where both point sets are bounded continua. We generalize his results by giving conditions which are both necessary and sufficient, and by removing the condition that both point sets be continua; see Theorems 16 and 17 . Theorem 18 is concerned with the separation of a disconnected subset of a continuum by a simple closed curve. The case where the point set $T$ mentioned in this theorem consists of one point is of particular interest, since it implies the existence of separation curves, for the case of a continuum which is disconnected by the omission of one of its points; see Theorems 21 and 23. Theorems such as 21 and 23 are often useful in proving the connectivity of point sets. The case where $T$ consists of one or two points is of particular interest in questions concerning the separation of unbounded point sets; see the statements and proofs of Theorems 20, 21, and 23.

We find useful the notion of one point set's being connected near another, and the notion of one point set's not being separated by another near a third point set. These concepts play a fundamental role in our treatment of separation theorems: in Theorems 6, 7, and 9 concerning conditions sufficient to make a point set strongly connected, and Theorems 12,14 , and 15 concerning the relation of a domain to its boundary. Theorems $4,11,13,22$, and 24 are concerned with conditions for accessibility.

THEOREM 1. If $M$ is a bounded maximal connected subset of a closed point set $K$ and $e$ is a positive number, then there exists a multiply connected Jordan domain $H$ containing $M$ such that (1) the boundary of $H$ contains no point of $K$, and (2) if $P$ is a point of $H^{\prime}$, then $d(P, M)<e . \dagger$

* See Concerning the separation of point sets by curves, loc. cit., Theorem 2.

$\dagger$ If $M$ is a point set, $S-M$ is the complement of $M$. A domain is a connected point set whose complement is closed. A Jordan domain or Jordan region is the interior of a simple closed curve. A 
Since $M$ is bounded, there exist at most a finite number, $n$, of bounded, mutually exclusive complementary domains* $D_{1}, D_{2}, D_{3}, \cdots, D_{n}$ of $M$ which contain points whose distance from $M$ is greater than $\frac{1}{2} e$. Let $D_{0}$ be the unbounded complementary domain of $M$. For each integer $i(i=0,1,2$, $3, \cdots, n)$, let $C_{i}$ be a circle, which, with its interior, is a subset of $D_{i}$; let $P_{i}$ be the center of $C_{i}$ and $L_{i}$ be a circle having the same center as $C_{i}$ and having an interior containing $M$. Let $N_{i}$ be the annular domain bounded by $C_{i}+L_{i}$. Then $N_{i}$ contains $M$. Let the equation $w_{0}=f_{0}(z)$ define the identity transformation of the plane into itself. For $i=1,2,3, \cdots, n$, let the equation $w_{i}=f_{i}(z)$ define an inversion of the plane about the point $P_{i}$ with reference to the circle $C_{i}$. If $Q$ is a point set in the $z$ plane, let $(Q)_{i}=f_{i}(Q)$.

For $i=0,1,2,3, \cdots, n, z=f_{i}^{-1}\left(w_{i}\right)$ is a uniformly continuous function of $w_{i}$ over the closed and bounded point set $\left(N_{i}\right)_{i}^{\prime}$. Hence, there exists a positive number, $d_{i}$, such that when $\left|w_{1_{i}}-w_{2_{i}}\right|<d_{i},\left|z_{1}-z_{2}\right|<\frac{1}{2} e$, where $w_{1_{i}}$ and $w_{2_{i}}$ are points of $\left(N_{i}\right)_{i}^{\prime}$. Let $m_{i}$ be the point set consisting of $(M)_{i}$ plus all bounded complementary domains of $(M)_{i}$. Let $k_{i}=m_{i}+(K)_{i}$.

If $m_{i}$ is not a maximal connected subset of $k_{i}$, there must exist a connected subset of $k_{i}$ which is the sum of $m_{i}$ and $t_{i}$, where $t_{i}$ is a point set containing no point of $m_{i}$. It is easily seen that both $k_{i}$ and $m_{i}$ are closed. Hence, if $T_{i}$ is a maximal connected subset of $t_{i}^{\prime}$, it must have a limit point in $m_{i}$. Since $T_{i}$ is a subset of the unbounded complementary domain of the closed point set $(M)_{i}, T_{i}^{\prime}$ must be a subset of $(K)_{i}$. Hence, $(M)_{i}+T_{i}$ is a connected subset of $(K)_{i}$. If $T_{i}$ is non-vacuous, then $M$ is not a maximal connected subset of $K$, contrary to the hypothesis of the theorem. Thus, $k_{i}$ is a closed point set, and $m_{i}$ is a bounded maximal connected subset of $k_{i}$, which does not separate space. Hence, there exists $\dagger$ a simple closed curve $j_{i}$, which encloses $m_{i}$, contains no point of $k_{i}$, and whose interior contains no point whose distance from $m_{i}$ is greater than $d_{i}$. From the definition of $j_{i}$ it follows that $j_{i}$ is a subset of $\left(D_{i}\right)_{i}$, and that no point of $\left(D_{i}\right)_{i}$ within $j_{i}$ is at a distance greater than $d_{i}$ from $(M)_{i}$. Let $J_{i}=f_{i}^{-1}\left(j_{i}\right)$. Then the domain $H$ bounded by the finite collection $\sum_{i=0}^{n} J_{i}$ satisfies the conclusion of the theorem.

multiply connected Jordan domain, of order $n$, is a domain whose boundary consists of $n$ mutually exclusive simple closed curves. In this paper we shall consider only domains of a finite order. Thus,a Jordan region is of order unity. If $M$ is a point set, by $M^{\prime}$ is meant the set of all limit points of $M$ and $M+M^{\prime}$ is denoted by $\bar{M}$. If $M$ and $N$ are point sets, by $d(M, N)$, the distance from $M$ to $N$, we mean the lower bound of the distances between pairs of points, $x$ and $y$, where $x$ is a point of $M$ and $y$ of $N$. $S-\bar{M}$.

* If $M$ is a point set, by a complementary domain of $M$ is meant a maximal connected subset of $\dagger$ Cf. Theorem 1 of Moore's paper Concerning the separation of point sets by curves. 
Theorem 2. If $K$ is a point set, $e$ is a positive number, and $L$ is a bounded point set which is either $K$ or a closed point set which is the sum of a collection of maximal connected subsets of $\bar{K}$, then there exists a finite collection of multiply connected Jordan domains covering $L$ and such that (1) the upper distance* of any of these domains from the product of $L$ and that domain is less than $e$, (2) the distance between any pair whatever of domains of this collection is a positive number, (3) the boundary of this collection of domains contains no point of $\bar{K}$, and (4) each domain contains a point of $L . \dagger$

For each maximal connected subset $A$ of $\bar{L}$ there exists, by Theorem 1 , a multiply connected Jordan domain $H_{A}$ such that no point of the boundary of this domain is a point of $\bar{K}$, and the upper distance of $H_{A}{ }^{\prime}$ from its product with $\bar{L}$ is less than $e / 6$. By the Heine-Borel-Lebesgue Theorem there exists a finite sub-collection $H_{1}$, which covers $\bar{L}$, of the collection $\left[H_{A}\right]$. Let $T$ be the set of all points which belong to elements of $H_{1}$. There exist then a finite number, $k$, of maximal connected subsets, $T_{1}, T_{2}, T_{3}, \cdots, T_{k}$, of $T^{\prime}$. Let $d$ be a positive number which is smaller than $e / 6$, and is also smaller than the smallest of the positive numbers $d\left(T_{i}, T_{j}\right) / 3$, where $i, j=1,2,3$, $\cdots, k$, and $i \neq j$. By an argument analogous to that used in a similar connection in the proof of Theorem 1 it can be shown that $T_{i}(i=1,2,3$, $\cdots, k)$ is a maximal connected subset of $\bar{T}+\bar{K}$; hence, by Theorem 1 , there exists a multiply connected Jordan domain $N_{i}$ covering $T_{i}$ such that no point of $N_{i}$ is at a distance from $T_{i}$ greater than $d$, and that the boundary of $N_{i}$ contains no point of $\bar{T}+\bar{K}$. Then $\sum_{i=1}^{k} N_{i}$ is a collection of domains satisfying the conclusion of the theorem.

Theorem 3. If $K$ is a closed point set, $L$ is a closed and bounded point set which is the sum of a collection of maximal connected subsets of $K, d$ is a positive number such that no maximal connected subset of $L$ has a diameter greater than $d$, and $e$ is any positive number whatever, then there exists a finite collection of Jordan domains covering $L$ such that (1) no domain in this collection has a diameter greater than $e+d ;(2)$ if $D$ and $\bar{D}$ are any two domains whatever in this collection, then $d(D, \bar{D})>0 ;(3)$ the boundaries of these domains have no

* By the upper distance from $M$ to $N$, written $u(M, N)$, we mean the upper bound of the set of values $[d(A, N)]$, where $A$ is a variable point of $M$. Cf. R. L. Moore, Concerning upper semi-continuous collections of continua, these Transactions, vol.27 (1925), p.416, second footnote. A collection $M$ of domains is said to cover a point set $K$, provided every point of $K$ belongs to some element of the collection $M$. The diameter of the point set $M$ is the upper bound of the set of values $[d(A, B)]$ where $A$ and $B$ are points of $M$.

† Theorems 2 and 3 are generalizations of results stated by Schoenflies and Kerékjárto. Cf. A. Schoenflies, Die Entwickelung der Lehre von den Punktmannigfaltigkeiten, Berlin, Teubner, 1908, pp. 104-106; and B. von Keréjárto, Vorlesungen ueber Topologie, Berlin, Springer, 1923, pp. 49-52 
points in common with $K$; (4) each domain contains a point of $L$. In particular, if the point set $L$ is totally disconnected, $\dagger$ the conclusion holds, if the number $d$ mentioned in the hypothesis is zero.

The interior of a simple closed curve has the same diameter as the curve itself. It follows by Theorem 2 that if the theorem is not true, there must exist a positive number $f$ and a sequence of positive numbers, $e_{1}, e_{2}, e_{3}, \cdots$, $e_{n}, \cdots$, such that (1) $e_{n}$ approaches zero as $n$ approaches infinity; (2) for each positive integer $n$ there exists a finite collection $H_{n}$ of multiply connected Jordan domains which have, with respect to the point sets $L$ and $K$ and the positive number $e_{n}$, the properties (1), (2), (3), and (4) mentioned in the conclusion of Theorem 2; (3) for each $n$ the collection $H_{n}$ contains a domain $h_{n}$ of diameter greater than $d+f$. It can readily be shown that the collection $\left[h_{n}\right]$ has an infinite sub-collection which has a closed and connected limiting set, $\ddagger$ and that this limiting set has a diameter not less than $d+f$. Since $L$ is closed, this limiting set is a subset of $L$. Hence the supposition that the theorem is not true leads to a contradiction of the hypothesis that $L$ has no connected subset of diameter greater than $d$.

Theorem 4. Given that $A$ and $B$ are distinct points and $G$ is a bounded collection of point sets such that (1) if $e$ is a positive number, there exist at most a finite number of elements of $G$ having a diameter greater than $e$; (2) if $G^{*}$ is the sum of the elements of $G$, and $g$ is an element of $G$, then $d\left(g, G^{*}-g\right)>0$; and (3) for each element $g$ of $G$ there exists a simple continuous arc whose end points are $A$ and $B$, and which contains no point in common with the point set $g$. Then there exists a simple continuous arc $A W B$ which contains no point in common with $G{ }^{*}$

It follows from condition (2) of the hypothesis that the elements of $G$ are countable and hence can be put in a one-to-one correspondence with the set of positive integers. Let $g_{1}$ be the first element of $G$ in this ordering, and let $h_{1}$ be a simple continuous arc which contains no point of $g_{1}$ and whose end points are $A$ and $B$. For $i$ an integer greater than unity, let $g_{i}$ be the first

$\dagger$ A point set is said to be totally disconnected if it has no connected subset containing more than one point.

$\ddagger$ Cf. S. Janiszewski, Sur les continus irréductibles entre deux points, Journal de l'École Polytechnique, (2), vol. 16 (1912), p. 97. By the limiting set of a collection $G$ of point sets we mean the set of all points $P$ such that every domain containing $P$ contains points of infinitely many elements of the collection $G$. Cf. Janiszewski, loc. cit., p. 93, last four lines; A. Schoenflies, Beiträge zur Theorie der Punktmengen, Mathematische Annalen, vol. 59 (1904), p. 139, paragraphs III and IV, and Bemerkung zu meinem zweitem Beitrag zur Theorie der Punktmengen, Mathematische Annalen, vol. 65 (1908), pp. 431-432. 
element in $G$ distinct from the elements in the collection $\sum_{j=1}^{i-1} g_{j}$ which contains points in common with $h_{1}$, and let $h_{i}$ be an arc whose end points are $A$ and $B$, and which contains no points in common with $g_{i}$. Let $F_{0}$ be a closed point set which contains no points in common with $\bar{G}^{*}$. If $i$ is a positive integer, it follows by part (2) of the hypothesis that if $g_{i}{ }^{*}$ is a maximal connected subset of $\bar{g}_{i}$, then it is a maximal connected subset of $\bar{G}^{*}$. Hence, by Theorem 2, there exists for each positive integer $i$ a finite collection $F_{i}=f_{i 1}$ $+f_{i 2}+\cdots+f_{i n_{i}}$ of multiply connected Jordan domains covering $g_{i}$ and such that (1) the upper distance of $F_{i}$ from $g_{i}$ is less than one half the smallest of the three positive numbers $1 / i, d\left(g_{i}, G^{*}-g_{i}\right)$, and $d\left(g_{i}, \sum_{j=1}^{i-1} F_{j}\right) ;(2)$ the distance between any two distinct elements of $F_{i}$ is a positive number; (3) the boundary, $B_{i}$, of $F_{i}$ contains no point of $\bar{G}^{*}$. Let $E$ be the set of all points of the arc $h_{1}$ belonging to $S-\sum_{i=1}^{\infty} \sum_{j=1}^{n_{i}} f_{i j}$; let $C_{i}$ be the set of all points common to $F_{i}$ and the $\operatorname{arc} h_{i}$; and let $\bar{K}=E+\sum_{i=1}^{\infty}\left(B_{i}+C_{i}\right)$. It may readily be seen that $\bar{K}$ is a closed, bounded point set containing no point of $G^{*}$. Let $K_{1}$ be the maximal connected subset of $\bar{K}$ containing the point $A$. If $K_{1}$ does not contain $B$ there exists, by Theorem 1, a simple closed curve $J$, containing no point of $\bar{K}$, such that one of the complementary domains of $J$ contains $A$ and the other contains $B$. Hence, $J$ contains no points in common with the point set $\sum_{i=1}^{\infty} B_{i}$ and thus either (1) must be a subset of some domain $f_{i j}$ of the collection $\sum_{i=1}^{\infty} \sum_{j=1}^{n_{i}} f_{i j}$ or (2) must be entirely without all the domains of this collection. The first case is impossible; for the arc $h_{i}$ contains the points $A$ and $B$, and thus contains a point $x_{i}$ on $J ; x_{i}$ belongs to $F_{i}$, and hence to $C_{i}$ and $\bar{K}$, thus contradicting the definition of $J$. Similarly, in the second case, $J$ must contain a point of $h_{1}$ which is also a point of $E$ and of $\bar{K}$. Thus, the supposition that $K_{1}$ does not contain $B$ leads to a contradiction.

Hence, $K_{1}$ is a closed, connected, bounded point set containing $A$ and $B$, but containing no point of $G^{*}$. It may readily be shown with the help of a theorem $\dagger$ due to R. L. Moore and a theorem $\ddagger$ of Sierpinski's that $K_{1}$ is connected im kleinen at all of its points. It follows that $K_{1}$ is a continuous curve and that there exists within $K_{1}$ a simple continuous arc whose end points are $A$ and $B . \S$

$\dagger \mathrm{Cf}$. Moore, Report on continuous curves from the viewpoint of analysis situs, Bulletin of the American Mathematical Society, vol. 29 (1923), pp. 296-297, \$3.

$\ddagger$ Cf. W. Sierpinski, Un thêorème sur les continus, Tôhoku Mathematical Journal, vol. 13 (1918), pp. $300-303$.

$\S$ Cf. R. L. Moore, $A$ theorem concerning continuous curves, Bulletin of the American Mathematical Society, vol. 23 (1917), pp. 233-236; S. Mazurkiewicz, Sur les lignes de Jordan, Fundamenta Mathematicae, vol. 1 (1920), pp. 196-209; and R. Tietze, Ueber stetige Kurven, Jordansche Kurvenbogen, und geschlossene Jordansche Kurven, Mathematische Zeitschrift, vol. 5 (1919), pp. 284-291. 
TheOREM 5. If $M$ is a closed and bounded point set, $G$ is the aggregate of $g$ 's, where the symbol $g$ represents a maximal connected subset of $M, K$ is a bounded continuum containing at least one point in common with each element of $G$, and for each $g, h_{o}$ is the point set $g \cdot(\overline{K-K \cdot M})$ and $H_{\theta}$ is a continuum containing $h_{\theta}$, then if $\left.\overline{\left(K-K \cdot M+\left[H_{\theta}\right]\right.}\right)$ is bounded, it is a continuum.

The theorem follows from Theorem 1 if $M$ is connected. We shall suppose that $G$ contains more than one element. Let $g$ be a definite element of $G$, and $e$ be a positive number which is less than $u(K, g)$. It can be shown with the help of Theorem 1 that there exist points of $K-K \cdot M$ within a distance of $e$ from $g$. It follows that $h_{g}$ is non-vacuous.

Let $N=\overline{\left(K-K \cdot M+\left[H_{0}\right]\right)}$. If $N$ is not connected, there exists, by Theorem 1, a simple closed curve $J$ having no points in common with $N$ and such that its interior $D_{1}$ and its exterior $D_{2}$ both contain points in common with $N$. If a point set $h_{i}$ contains points in one of these domains, evidently $H_{i}$, and therefore $h_{i}$, is a subset of that domain. Let $M_{1}$ and $M_{2}$ respectively be the sums of all those elements $g$ of $G$ for which the corresponding $H_{0}$ 's are subsets of $D_{1}$ and $D_{2}$ respectively. The point set $M_{1}$ is closed. For, let $z$ be a limit point of $M_{1}$ which does not belong to $M_{1}$. Since the elements of $G$ are closed, there must exist an infinite sub-collection $\bar{G}_{1}$ of elements of $G$, such that (a) the sum of the elements of $\bar{G}_{1}$ is a subset of $M_{1}$, (b) every infinite sub-collection of $\bar{G}_{1}$ has a limiting set containing $z$, and (c) no element of $\bar{G}_{1}$ contains $z$. The limiting set $F$ of $G_{1}$ is closed and connected. $\dagger$ It follows that $F$ is a subset of an element of $G$. Let $\bar{h}_{1}$ be the collection of the $h_{i}$ 's corresponding to the elements of $\bar{G}_{1}$. We have shown that this collection is non-vacuous. The limiting set of $h_{1}$ is evidently a subset of $F$ and of $D_{1}$. But this limiting set is also a subset of the $h$ of that element of $G$ which contains $F$, since every point in it is a limit point of $K-K \cdot M$. Hence $F$ is a subset of an element of $\bar{G}_{1}$. It follows that $M_{1}$ and $M_{2}$ are closed and mutually separated.

Let $K_{1}$ and $K_{2}$ be those subsets of $(K-K \cdot M)$ which are subsets of $D_{1}$ and $D_{2}$ respectively. Since an " $h$ " corresponding to an element of $\bar{G}_{1}$ is a subset of $D_{1}, K_{2}$ can have no point or limit point in $M_{1}$. By definition $M_{1}$ can have no limit point in $K_{2}$. It may readily be seen that the point sets $\overline{\left(M_{1}+K_{1}\right)}$ and $\overline{\left(M_{2}+K_{2}\right)}$ are mutually separated. But this contradicts the fact that

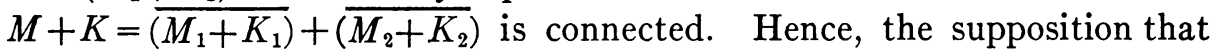
$N$ is not connected has led to a contradiction.

Definitions. If $K, M_{1}$, and $M_{2}$ are point sets, $K$ has no points in common with $M_{1}+M_{2}$ and no connected subset of $S-K$ contains points of both $M_{1}$

† Cf. Janiszewski, loc. cit. 
and $M_{2}$, then we say that $M_{1}$ and $M_{2}$ are separated by $K$ or that $K$ separates $M_{1}$ from $M_{2}$. If $K$ and $M$ are mutually exclusive point sets and $M$ is not a subset of any connected subset of $S-K$, then we say that $K$ separates $M$. If $K, H$, and $T$ are point sets, $H$ and $K$ are mutually exclusive, and for every positive number $e$ and every point $P$ of $T$ there exists a positive number $d_{e P}$ such that any two points of $K$ whose distance from $P$ is less than $d_{e P}$ can be joined by a connected subset $h_{e P}$ of $S-H$, whose upper distance from $P$ is less than $e$, then we say that $K$ is not separated by $H$ near $T$. $\dagger$ If $K$ and $T$ are point sets, then $K$ is said to be connected near $T$, provided that for every point $P$ of $T$ and every positive number $e$ there exists a positive number $d_{e P}$, such that any two points of $K$ whose distances from $P$ are each less than $d_{e P}$ are subsets of a connected point set $h_{e P}$ which is a subset of $K$ and which is at an upper distance less than $e$ from $P$. If, in the preceding two definitions, it be specified that the connected point set $h_{e P}$ be a continuum, then we say that $K$ is strongly not separated by $H$ near $T$, and $K$ is strongly connected near $T$, respectively. If there exists for each positive number $e$ a positive number $d_{e P}$, which, for the case of the definitions given in the preceding sentences, is independent of $P$, then we say respectively that $K$ is uniformly not separated by $H$ near $T$, and $K$ is connected near $T$ uniformly. If the point set $T$ is closed and bounded, then, by an argument similar to that used in proving that a function which is continuous over a closed and bounded point set is uniformly continuous over that point set, it may be shown that if $K$ is connected near $T$, or is not separated by $H$ near $T$, then it has these properties uniformly over $T$. If $H$ is closed and $K$ is not separated by $H$ near $T$, then $K$ is strongly not separated by $H$ near $T$; but this conclusion does not follow if $H$ is not closed, as the following example will show. For $0 \leqq x \leqq 1$ let $A_{x}$, $B_{x}, C_{x}, D_{x}$, and $E_{x}$ be the points with coördinates $(0, x),(1, x),(x, 0),(x, 1)$, and $(x, 2)$ respectively; and let $K=A_{0} B_{0}+C_{0} D_{0} E_{0}+\sum_{i=1}^{\infty} C_{1 / i} D_{1 / i} E_{1 / i}$, and let $H=S-\left(K-A_{0}\right)$. Then $K-A_{0}$ is not separated by $H$ near $A_{0}$, but it is not true that $K-A_{0}$ is strongly not separated by $H$ near $A_{0}$. Furthermore, $K-A_{0}$ is connected near $A_{0}$, but it is not true that $K-A_{0}$ is strongly connected near $A_{0}$.

If $K$ is connected near $T$, and $T=K$, then $K$ is everywhere connected im kleinen. If $K$ and $H$ are mutually exclusive, and $T$ is any subset of $S-\bar{H}$, then $K$ is not separated by $H$ near $T$. If $K$ is connected near $T$, and $K$ and $H$ are mutually exclusive, then $K$ is not separated by $H$ near $T$. If $K$ is not separated by $H$ near $T$, then neither is any subset of $K$.

† Zarankiewicz, Sur les coupures locales faites par les continus, Bulletin de l'Académie Polonaise des Sciences et des Lettres, Classe des Sciences Mathématiques et Naturelles, Série A: Sciences Mathématiques, 1927, p. 194, defines a concept with some similarity to ours but less general. 
Theorem 6. If $K$ is a bounded continum, $T$ is a closed subset of the boundary of $S-K, T$ does not separate $K-T$, and $S-K$ is connected near $T$, then $K-T$ is strongly connected.

If the theorem is not true, there must exist a pair of points $x$ and $y$ of $K-T$, such that any closed subset of $K-T$ containing $x$ and $y$ is not connected. Since $T$ is closed, it can be proved, with the help of Theorems 10 and 15 of the paper F. A., that there exists a simple continuous arc xty containing no point of $T$. Let $0<e<\frac{1}{2} d(x t y, T)$. Since $T$ is closed, and $S-K$ is connected near $T, S-K$ has this property uniformly over $T$; and there exists a positive number $d$ less than $e$, such that if $P$ is a point of $T$, and $x_{1}$ and $x_{2}$ are a pair of points of $S-K$ whose distances from $P$ are each less than $d$, then $x_{1}$ and $x_{2}$ belong to a connected subset of $S-K$ whose upper distance from $P$ is less than $e$.

By Theorem 2 the point set $T$ can be covered by a finite collection $H=h_{1}+h_{2}+h_{3}+\cdots+h_{k}$ of multiply connected Jordan domains, such that if $m$ and $n$ are distinct positive integers then (1) $T$ contains no point of the boundary of $h_{m}$, (2) no point of $h_{m}$ is at a distance from $T \cdot h_{m}$ greater than $d / 10,(3)$ the distance between $h_{m}$ and $h_{n}$ is positive. Let $K^{*}$ be the set of all points belonging to $K$ which are not covered by this collection of domains. The point set $K^{*}$ is closed. Let $X$ be the maximal connected subset of $K^{*}$ containing the point $x$. Clearly $X$ does not contain $y$. By Theorem 1 there exists a simple closed curve $J_{1}$ which separates $x$ from $y$, and contains no point of $K^{*}$. Suppose that the interior, $D_{1}$, of this curve, contains $y$, and that its exterior, $E_{1}$, contains $x$. In the order $x t y$ on the arc $x t y$ let $v$ be the first point common to $K$ and $D_{1}$; in the order $v x$ on the interval $v x$ of this arc let $z$ be the first point common to $J_{1}$ and $x t y$, and $w$ be the first point common to this interval and the product of $K$ and $E_{1}$. Let $z_{1}$ be a point in the order $w z_{1} z$ such that the interval $w z_{1}$ of $w z v$ contains no point of $J_{1}$. It is easily shown that there exists a simple closed curve $z_{1} q_{1} z q z_{1}$ which has in common with the arc $x t y$ the two points $z$ and $z_{1}$, encloses the segment $z_{1} z$ of $x t y$, but encloses or contains no other points of $x t y$ or of $K$. Let $D$ be the complementary domain containing $v$ of the sum of the simple closed curves $J_{1}$ and $z_{1} q_{1} z q z_{1}$. Its boundary $J$ is a simple closed curve. $\dagger$ Then $x$ is in the exterior

† J. Pál in Om Planeus Topologi, Matematisk Tidskrift B, 1923, pp. 66-72 (Festskrift til J. Hjelmslev), states the following theorem: If the (simple, closed, plane) Jordan curves $a$ and $b$ have at least two points in common, then every complementary domain of their sum is bounded by a simple closed curve. This result, while not explicitly stated by R. L. Moore, is easily derived from theorems he has proved; cf. either F. A., Theorem 41, p. 155, or On the Lie-Riemann-Helmholtz-Hilbert problem of the foundations of geometry, American Journal of Mathematics, vol. 41, pp. 299-319, Theorem 26. 
$E$ of $J, J$ contains in common with $w z v$ only the point $z$, and contains no points of $K^{*}$. Also, the arc wzv contains in common with $K$ only the points $w$ and $v$.

Let $K_{i}$ be the product of $h_{i}^{\prime}$ and $K$. Let $z_{2}$ and $z_{3}$ be two distinct points of $J$ distinct from $z$. In the order $z z_{2} z_{3}$ on $J$ let $A$ be the first point common to $J$ and $K_{1}$, and let $B$ be the first point on $J$ in the order $z z_{3} z_{2}$ common to $J$ and $K_{1}$. Let $A z B$ be the interval $A z B$ of $J$. Since $h_{1}^{\prime}$ is connected, there exists a finite chain of points $x_{1}(=A), x_{2}, x_{3}, \cdots, x_{m}(=B)$ belonging to $h_{1}^{\prime}$, such that the distance between any two consecutive points in this sequence is less than $d / 10$. Let $T_{i}$ be the product of $h_{i}$ and $T$. From the definition of $h_{1}$ it follows that, for every value of $i$ in the sequence $1,2,3, \ldots, m$ within a distance $d / 10$ of $x_{i}$ there exists a point $y_{i}$ of $T_{1}$. Furthermore, since $T_{1}$ belongs to the boundary of $S-K$, there exists, within a distance $3 d / 10$ of $y_{i}$, a point $t_{i}$ belonging to $S-K$. It is possible to select $t_{1}$ and $t_{m}$ in such a way that $t_{1}$ belongs to the segment $A z$ of the $\operatorname{arc} A z B$, and that $t_{m}$ belongs to the segment $z B$ of this arc; for, since the curve $J$ contains no points in common with $K^{*}$, the points $A$ and $B$ are limit points of the product of $A z B$ and $S-K$.

For $i=1,2,3, \cdots, m-1$, the points $t_{i}$ and $t_{i+1}$ are each at a distance less than $d$ from the point $y_{i}$ of $T_{1}$. There exists a connected subset $\left(t_{i}\right.$, $\left.t_{i+1}\right)$ of $S-K$, whose upper distance from $y_{i}$ is not greater than $e$. Then $F=\sum_{i=1}^{m-1}\left(t_{i}, t_{i+1}\right)$ is a connected subset of $S-K$ and its distance from the arc $v z w$ is greater than $e$. It can be proved with the help of Theorems 10 and 15, F. A., that there exists a simple continuous $\operatorname{arc} t_{1} \bar{t}_{m}$ which is a subset of $S-K$ and contains no point whose distance from vzw is less than $\frac{1}{2} e$. The sum of the interval $t_{1} z t_{m}$ of $J$ and the arc $t_{1} \bar{t}_{m}$ has as a subset a simple closed curve $C_{1}$ which (1) has no point in common with $K^{*}+K_{1}$, (2) has an interval $E_{1} z F_{1}$ in common with $J$, and (3) has in common with the arc vzw the point $z$ and this point only. By a similar argument, this time using the curve $C_{1}$ instead of $J$, we can show the existence of a simple closed curve $C_{2}$ which has no point in common with $K^{*}+K_{1}+K_{2}$ and which has the properties (2) and (3) above. If we continue this process we get after $k$ steps a simple closed curve $C_{k}$ which (1) contains no point in common with $K=K^{*}$ $+\sum_{i=1}^{k} K_{i}$, (2) has an interval $E_{k} z F_{k} \dagger$ in common with $J$, (3) has in common with the arc vzw the point $z$ and the point $z$ only. Since the arc vzw intersects $\ddagger$ the $\operatorname{arc} E_{k} z F_{k}$ at $z, v$ is in one complementary domain of $C_{k}$ and $w$ is in the other. Since $C_{k}$ has no point in common with $K$, and $K$ is connected, we have a contradiction. Hence, $K-T$ is strongly connected.

\footnotetext{
† Our notation implies that $z$ is not an end point of $E_{k} z F_{k}$.

$\ddagger$ We shall say that a point set $A_{1}$ intersects a point set $A_{2}$ at a point $P$ provided that $A_{1}-A_{1} \cdot A_{2}$ is separated by $A_{2}$ near $P$.
} 
TheOREM 7. If $T$ is a closed subset of a bounded continuum $K, K-T$ is connected, and $K-T$ is strongly connected near $T$, then $K-T$ is strongly connected. $\dagger$

Let $x$ be a point of $K-T$. Let $X$ be the set of all points of $K-T$ which can be joined to $x$ by a closed and connected subset of $K-T$. Let $Y=K-T-X$. If $Y$ is vacuous, the theorem must be true. If $Y$ is non-vacuous, there must exist a point $z$ which belongs to one of the point sets $X$ and $Y$, and is a limit point of the other.

Suppose, first, that $z$ belongs to $X$. Then $z$ is the sequential $\ddagger$ limit point of a sequence of points $y_{1}, y_{2}, y_{3}, \cdots$ belonging to $Y$. For each integer $i$ let $h\left(y_{i}\right)$ be the set of all points in $K-T$ which can be joined to $y_{i}$ by a continuum which is a subset of $K-T$. The point set $h\left(y_{i}\right)$ must have at least one limit point in $T . \S$

By a theorem $\|$ of Janiszewski's the limiting set $L$ of $\sum_{i=1}^{\infty} h\left(y_{i}\right)$ is closed and connected. Since $T$ is closed and bounded, $K-T$ is strongly connected near $T$, uniformly. Hence, if $e=\frac{1}{2} d(z, T)$, there exists a positive number $d$ less than $e$, such that if $P$ is a point of $T$, and $w_{1}$ and $w_{2}$ are two points of $K-T$, each of whose distances from $P$ is less than $d$, then there exists a closed and connected subset of $K-T$ containing $w_{1}$ and $w_{2}$ whose upper distance from $P$ is less than $e$. Let $L_{1}$ be the maximal connected subset containing $z$ of the point set consisting of all points of $L$ whose distance from $T$ is not less than $\frac{1}{2} d$. By an argument similar to that used in the preceding paragraph it follows that $L_{1}$ is closed, connected, and contains a point $v_{1}$ whose distance from $T$ is $\frac{1}{2} d$. As $v_{1}$ belongs to the limiting set of $\sum_{i=1}^{\infty} h\left(y_{i}\right)$, there must exist an integer $j$ such that $h\left(y_{j}\right)$ contains a point $v_{2}$ whose distance from $v_{1}$ is less than $\frac{1}{2} d$. There exist in $K-T$ three continua, $k_{1}, k_{2}$, and $k_{3}$, such that $k_{1}$ contains $v_{1}$ and $v_{2}, k_{2}$ contains $v_{2}$ and $y_{j}$, and $k_{3}$ contains

$\dagger$ The conclusions of this theorem and of Theorem 9 do not follow if the word "strongly" be omitted from the statement " $K-T$ is strongly connected near $T$, " as the following example will show. Let $K$ be the point set $K$ mentioned in the paragraph preceding the statement of Theorem 6, and let $T$ be the point $A_{0}$ there mentioned. Note, however, that this point set satisfies the hypothesis of Theorem 8.

The condition that $K-T$ be connected near $T$ is not necessary for the conclusion of Theorem 7 . Consider the set $K$ mentioned above, but let $T$ be the point $E_{0}$. Then $K-T$ is strongly connected, but is not connected near $T$.

$\ddagger$ The point $P$ is the sequential limit point of a sequence $P_{1}, P_{2}, P_{3}, \cdots$ of points, provided that every domain containing $P$ contains all except a finite number of the points of the given sequence. Cf. F. A., p. 134.

§ Cf. R. L. Wilder, Concerning continuous curves, Fundamenta Mathematicae, vol. 7, p. 372, lines 17-33.

|| Cf. Janiszewski, loc. cit. 
$z$ and $x$. Then $k_{1}+k_{2}+k_{3}+L_{1}$ is a continuum which contains $x$ and $y_{j}$, and is a subset of $K-T$. This contradicts the definition of $y_{j}$. Hence $z$ must belong to $Y$.

Hence there exists a sequence $x_{1}, x_{2}, x_{3}, \cdots$ of points belonging to $X$ having $z$ as a sequential limit point. For each positive integer, $i$, there exists in $X$ a continuum $l_{i}$ containing both $x_{i}$ and $x$. Let $L_{2}$ be the limiting set of $\sum_{i=1}^{\infty} l_{i}$. If $L_{2}$ contains no points of $T$, then there exists in $S-K$ a continuum $L_{2}$ containing $x$ and $z$, contrary to the fact that $z$ belongs to $Y$. If $L_{2}$ contains points in common with $T$, we get a contradiction precisely as in the preceding paragraph. Thus $Y$ is vacuous, and $K-T$ is strongly connected.

THEOREM 8. If $T$ is a set of condensation of a bounded continuum $K$, and $K-T$ is connected near $T$, then $K-T$ is connected. $\dagger$

If $K-T$ is not connected it is the sum of two mutually separated, nonvacuous point sets $M$ and $N$. Then, since $K$ is a continuum, $\bar{M}$ contains a limit point $z$ of $K-\bar{M}$. Every point of $T$ belonging to $K-\bar{M}$ is a limit point of $N$. Since $K-\bar{M}$ is a subset of $N+T, z$ is a point or a limit point of $N$; also, $z$ is a point or limit point of $M$. It follows from the hypothesis that there exists a circle about $z$, the interior of this circle containing a point of $M$ and a point of $N$, these two points being joined by a connected subset of $K-T$. Thus the supposition that $K-T$ is not connected has led to a contradiction.

Theorem 9. If $T$ is a closed set of condensation of a bounded continuum $K$, and $K-T$ is strongly connected near $T$, then $K-T$ is strongly connected.

This theorem is a consequence of Theorems 8 and 7.

Theorem 10. Given that $P$ is a point on the boundary $H$ of a domain $D$, and that there exists a circle $(\bar{K})$ with center at $P$, and an infinite collection $D_{1}$, $D_{2}, D_{3}, \cdots$ of mutually exclusive maximal sub-domains of the product of $D$ and the interior of $(\bar{K})$, and that $P$ belongs to the limiting set of $D_{1}, D_{2}, D_{3}$, ... Then if $(C)$ and $(K)$ are two circles concentric with such a circle, $(C)$ is within $(\bar{K})$, and $(K)$ is within $(C)$, there exists on $(C)$ an interval $A E$ and on $(K)$ an interval $B F$ such that (1) there exists on the interval $A E$, in the order indicated, an infinite sequence $A_{1}, A_{2}, A_{3}, \cdots$ of points having $A$ as a sequential

$\dagger$ This theorem was suggested and proved by Dr. H. M. Gehman during a discussion of the proof of my Theorem 9. A point set $T$ is said to be $a$ set of condensation of a point set $K$, if every point of $T$ is a limit point of $K-K \cdot T$. If $K$ is closed, $K$ contains $T$. Janiszewski, loc. cit., p. 102, defines in an analogous manner a continuum of condensation. 
limit point, and on the interval $B F$, in the order indicated, an infinite sequence $B_{1}, B_{2}, B_{3}, \cdots$ of points having $B$ as a sequential limit point; (2) for each positive integer $i$ there exists a simple continuous arc $A_{i} B_{i}$ which is a subset of $D_{i}$, and contains in common with $(C)$ only the point $A_{i}$ and in common with $(K)$ only the point $B_{i}$; (3) if $A_{i} A_{i+1}$ and $B_{i} B_{i+1}$ are the intervals $A_{i} A_{i+1}$ and $B_{i} B_{i+1}$, respectively, of $E A$ and $F B$, respectively, $J_{i}$ is the simple closed curve $A_{i} A_{i+1} B_{i+1} B_{i} A_{i}$, and $E_{i}$ is the interior of this curve, then $E_{i}$ is a subset of the annular domain bounded by $(C)$ and $(K)$ and if $i \neq j$, then $E_{i}$ and $E_{j}$ have no points in common; (4) for each $i$ there exists a sub-continuum $H_{i}$ of $H$, such that $H_{i}$ is a subset of $E_{i}^{\prime}$, and contains at least one point in common with $A_{i} A_{i+1}$ and at least one point in common with $B_{i} B_{i+1} ;(5)$ the collections $\sum_{i=1}^{\infty} H_{i}$ and $\sum_{i=1}^{\infty} A_{i} B_{i}$ have a common sequential limiting set $L$, which is a sub-continuum of $H$; (6) no two elements in the collection $\sum_{i=1}^{\infty} A_{i} B_{i}$ can be joined by a connected subset, which is entirely within $(\bar{K})$, of the domain $D ;(7)$ if $x$ and $y$ are points of two distinct elements of the collection $L+\sum_{i=1}^{\infty} H_{i}$, then there exists no connected subset of $S-D$ lying wholly within the annular domain bounded by $(C)$ and $(K)$, and containing both $x$ and $y$.

We leave the proof of this theorem to the reader; we refer him in particular to Theorem 1, and to an argument used by $\mathrm{R}$. L. Wilder $\dagger$ in another connection.

THEOREM 11. If $P$ is a point on the boundary of a domain $D$, then a necessary and sufficient condition that $P$ be accessible from $D$ is that there exist a subset $K$ of $D$ which is connected near $P$ and has $P$ as a limit point.

Obviously the condition is necessary. We will proceed to show that it is sufficient. It follows from the hypothesis that there exists a sequence of points $x_{1}, x_{2}, x_{3}, \cdots$ belonging to $K$ such that $P$ is the sequential limit point of this sequence, and such that for each positive integer $i$ there exists a connected subset of $K$ containing both $x_{i}$ and $x_{i+1}$, the upper distance of this connected subset from $P$ being less than $1 / i$. It follows by Theorems 10 and 15, F. A., that there exists a simple continuous arc $x_{i} x_{i+1}$, whose upper distance from $P$ is less than $2 / i$, and which is a subset of $D$. It follows by an

$\dagger$ Loc. cit., pp. 343-346.

$\ddagger$ The point $P$ on the boundary of a domain $D$ is said to be accessible from that domain provided that for every point $x$ of $D$ there exists a simple continuous arc $x P$, whose end points are $x$ and $P$, and which, except for the point $P$, is a subset of $D$. If for any simple continuous arc $A B$ whatever, such that the segment $A B$ is a subset of $D$, the point $P$ is accessible from every maximal domain of $D-D \cdot A B$, which has $P$ on its boundary, then $P$ is said to be accessible from all sides from $D$. Cf. Schoenflies, Entwickelung, loc. cit., p. 176. 
argument given by Wilder $\dagger$ and Theorem 15, F. A., that $P$ is accessible from D.

TheOREm 12. If $B$ is the boundary of a domain $D, P$ is a point of $B$, and $D$ is not connected near $P$, then either (1) the hypothesis of Theorem 10 is satisfied at $P$ with respect to the domain $D$ and its boundary, or (2) the point set $B-P$ is not connected, and there exists a simple closed curve $J$ which contains $P$, separates $B-P$, and, except for the point $P$, is a subset of $D$.

Suppose that the hypothesis of Theorem 10 is not satisfied. Then if $M$ is any circle about $P$, there exists a circle $N^{*}$ with the same center such that there exist at most a finite number of maximal sub-domains of the product of $D$ and the interior of $M$ containing points within $N .^{*}$ Hence at least one of these domains contains $P$ on its boundary. Let $H_{1}$ be such a domain with respect to a definite circle $J_{1}$ having its center at $P$, and $H_{0}=D$. It is impossible for $H_{1}$ and a circle $Q$ within $J_{1}$ and concentric with it to satisfy the hypothesis of Theorem 10 at $P$; for, if they did, $D$ and $Q$ would do the same, and this involves a contradiction of our hypothesis. As above, it follows that the product of $H_{1}$ and the interior of $Q$ has at least one subdomain having $P$ on its boundary. By a continuation of this process it is possible to select a sequence $J_{1}, J_{2}, J_{3}, \cdots$ of circles with centers at $P$ and with radii approaching zero monotonically as $n$ approaches infinity, and such that for each $n$ there exists a maximal sub-domain $H_{n}$ of the product of $H_{n-1}$ and the interior of $J_{n}$ having $P$ on its boundary. Let $y_{n}$ be a point of $H_{n}$ such that if $i \neq j$ then $y_{i} \neq y_{j}$. There exists in $H_{n}$ a simple continuous arc $\ddagger$ joining $y_{n}$ and $y_{n+1}$. It follows by Theorem 11 that there exists a simple continuous arc $y_{1} t P$ which has end points $y_{1}$ and $P$, except for $P$ is a subset of $D$, and for every $n$ has in common with $H_{n}$ a segment having $P$ as an end point.

Consider the following proposition: There exist two integers $i$ and $j, i$ a positive integer and $j$ a positive integer or zero, such that $H_{j}$ and the interior of $J_{i}$ have in common at least two maximal sub-domains each of which has $P$ on its boundary. Suppose the proposition is false, and let $N$ be a definite value of $j$ and $e$ be a positive number. Let $m>N$ be so chosen that $u\left(H_{m}, P\right)<e$. If $H_{N}-H_{m}$ had $P$ as a limit point, it would follow from the facts established at the beginning of the proof that $H_{N}-H_{m}$ and the interior of $J_{m}$ would have in common a maximal sub-domain having $P$ as a boundary point, and thus that $H_{N}$ and the interior of $J_{m}$ would contain two such domains; this contradicts the assumption that the proposition stated at the beginning of this

† Loc. cit., pp. 343-344, Case 1.

$\ddagger$ Cf. F. A., Theorem 15 . 
paragraph is false. It follows that there exists a circle with center at $P$ and containing no points of $H_{N}-H_{m}$. Since $H_{m}$ is a connected subset of $H_{N}$ and its upper distance from $P$ is less than $e$, where $e$ is independent of $N$, it follows that $H_{N}$ is connected near $P$. But this involves a contradiction of our hypothesis that $H_{0}=D$ is not connected near $P$.

It follows that there exists an arc $y_{1} w P$ of which $y_{1}$ and $P$ are the end points such that $y_{1} w P-P \subset D$, the product of $y_{1} t P$ and $y_{1} w P$ is their end points, and that for $n$ sufficiently large $y_{1} w P$ contains no point of $H_{n}$. Let $N$ be such an integer, and $J$ be the sum of $y_{1} t P$ and $y_{1} w P$. Then $J$ is a simple closed curve. There exists a Jordan domain $F_{N}$ which is a common subset of the interiors of $J_{N}$ and $J$, and whose boundary is a subset of $J_{N}+J$ and contains $P$. It is easily seen that the boundary of $F_{N}$ contains points in common with each of the segments $y_{1} t P$ and $y_{1} w P$ of $J$. Hence, since $F_{N}$ is connected and is not a subset of $D$, it must contain a boundary point of $D$. Similarly, the exterior of $J$ contains points of $B$. The truth of the theorem is thus established.

THEOREM 13. If a point on the boundary of a domain is not accessible from all sides from that domain, then the hypothesis of Theorem 10 is satisfied at that point.

Given a domain $D_{1}$, whose boundary $H_{1}$ contains a point $P$ which is not accessible from all sides from $D_{1}$. Then there must exist a pair of points $A$ and $B$ belonging to $\bar{D}_{1}$, a simple continuous arc $A X B$, which, except for $A$ and $B$, is a subset of $D_{1}$, and a sub-domain $D$ of $D_{1}$ whose boundary $H$ contains $P$, and is a subset of $H_{1}+A X B$, the point $P$ being not accessible from $D$. It is easily seen that all of the segment $A X B$ belongs to $H$, and that if $P$ is either $A$ or $B$ then $P$ is accessible from $D$. Suppose that $P$ is not a point of $A X B$. Then there exists a circle $K_{1}$ enclosing $P$ but no point of $A X B$. It can be shown, with the help of Theorems 11 and 12, that there exists within $K_{1}$ a circle $(\bar{K})$ which has with reference to $P, H$, and $D$ the properties mentioned in Theorem 10. It is easily seen that these properties hold also with reference to $P, H_{1}$, and $D_{1}$.

THEOREM 14. In order that a bounded domain be connected near its boundary it is necessary and sufficient that it be uniformly connected im kleinen.

It is easily seen that if a domain is uniformly connected im kleinen, then it is connected near its boundary. Let $D$ be a bounded domain which is connected near its boundary, $B$. If $D$ is not uniformly connected im kleinen, there must exist a positive number $e$ and an infinite sequence of pairs of points, $x_{1}, y_{1}, x_{2}, y_{2}, x_{3}, y_{3}, \cdots, x_{n}, y_{n}, \cdots$, such that if $n$ is any positive 
integer, then $d\left(x_{n}, y_{n}\right)<1 / n$, but $x_{n}$ and $y_{n}$ cannot be joined by a connected subset of $D$, of diameter less than $e$. The set of $x$ 's has a limiting set containing at least one point, $P$. Evidently $P$ belongs to $B$. There exists a number $d_{e P}$ such that if $x$ and $y$ are both points of $D$ at a distance less than $d_{e P}$ from $P$, then $x$ and $y$ both belong to a connected subset of $D$, whose upper distance from $P$ is less than $e / 3$. Also, there exists an integer, $n$, such that the distances of $x_{n}$ and $y_{n}$ from $P$ are each less than $d_{e P}$. Thus, the supposition that $D$ is not uniformly connected im kleinen has led to a contradiction.

THEOREM 15. In order that a domain be a Jordan domain it is necessary and sufficient that it be simply connected, $\dagger$ bounded, and connected near its boundary.

The theorem follows from Theorem 14 and a theorem by R. L. Moore. $\ddagger$

Theorem 16. If $K$ is a bounded continuum, $D$ is a complementary domain of $K, H$ is a bounded subset of $D$, and $\bar{H}=H+T$, where $T$ is a totally disconnected subset of $K$, then in order that there exist a simple closed curve, $J$, containing $T$, and separating $K-T$ from $H$, and such that $J-T$ is a subset of $D$, it is necessary and sufficient that $K$ should not separate $H$ near $T$.

We shall first prove the sufficiency of the condition. Consider the case where $\bar{H}$ is a continuum, and $H$ is connected near $T$. Since $T$ is closed and bounded, $H$ is connected near $T$, uniformly over $T$. It follows, with the help of Theorem 3, that for each positive integer $n$ there exist a pair of point sets $C_{n}$ and $E_{n}$ defined as follows: $C_{1}$ is a circle, whose interior $E_{1}$ contains $K+H$; for $n$ greater than unity $E_{n}$ consists of the sum of a finite collection of Jordan domains covering $T$ such that (1) each domain of this collection contains at least one point of $T$, and its diameter is less than $1 / n$; (2) the distance between any two domains in this collection is a positive number; (3) the boundary, $C_{n}$, of $E_{n}$ contains no point of $T$; (4) each domain of $E_{n}$ plus its boundary is a subset of exactly one domain of $E_{n-1} ;(5)$ if $t_{1}$ and $t_{2}$ are points of $H$ in or on the boundary of a maximal domain $D^{*}$ of $E_{n}$, then there exists within that domain of $E_{n-1}$ containing $D^{*}$ a connected subset $t_{1} t_{2}$ of $H$ containing $t_{1}$ and $t_{2}$. If $P$ is a point of $T$, let $D(n, P)$ be that maximal domain of $E_{n}$ which contains $P$, and let $J(n, P)$ be the boundary of $D(n, P)$. Let $\bar{x}$ and $\bar{y}$ be definite points of $K-T$ and $H$ respectively. Let $m$ be an integer greater than 10 such that $E_{m}^{\prime}$ contains neither $\bar{x}$ nor $\bar{y}$. Let $K_{1}{ }^{*}$ be the sum

$\dagger$ A domain is said to be simply connected if its boundary is connected.

$\ddagger$ Cf. R. L. Moore, A characterization of Jordan regions by properties having no reference to their boundaries, Proceedings of the National Academy of Sciences, vol. 4 (1918), pp. 364-370. 
of all those complementary domains of $K_{1}=K+\sum_{i=m}^{\infty} C_{i}$ which have no limit points in $H$. Let $K^{*}=\left(\overline{K_{1}{ }^{*}+K}\right)-T$.

No limit point of $K^{*}$ belongs to $H$. For, suppose $H$ contains such a limit point $Q$. Then there exists a Jordan domain $q$ containing $Q$ but having no point or limit point in common with $K$, and containing points of not more than one simple closed curve belonging to the collection $\sum_{i=1}^{\infty} C_{i}$. If $Q$ belongs to no such curve we may suppose that $q$ contains no point of $K_{1}$. Then $Q$ is a limit point of $K_{1}^{*}$. If $q$ is a subset of some domain which is a subset of $K_{1}^{*}$, this domain has a limit point in $H$, contrary to its definition. If $q$ contains points of at least two such domains, it must contain a boundary point of one of these domains, contrary to the hypothesis that $q$ contains no point of $K_{1}$. Hence $Q$ must belong to some simple closed curve $J_{1}$ of some $C_{i}$ of the collection $\sum_{i=1}^{\infty} C_{i}$. Suppose again that $q$ contains no point of $K$ or of any simple closed curve of some $C_{i}$ of the collection $\sum_{i=1}^{\infty} C_{i}$, distinct from $J_{1}$. Then there exists within $J_{1}$ a Jordan region $R_{1}$ and without $J_{1}$ a region $R_{2}$ such that the boundaries of these regions have in common a segment $t_{1} Q t_{2}$ of $J_{1}$, containing the point $Q$, and such that $R_{1}$ and $R_{2}$ are subsets of $q$. It follows that either $R_{1}$ or $R_{2}$ must contain points of $K_{1}^{*}$. But, by an argument used above, it can be shown that this is impossible. Since all limit points of $H$, except those belonging to $T$, belong to $H$, the argument just given shows that $K^{*}$ contains no points or limit points of $H$. Hence $H$ and $K^{*}$ are mutually separated. It is easily seen that $\bar{K}^{*}=K^{*}+T$ is a continuum.

By Theorem $8, H$ is connected. Let $D^{*}$ be that complementary domain of $K^{*}$ which contains $H$, and let $K_{2}=S-D^{*}$. It is easily seen that $K_{2}$ contains $K^{*}$ and is connected.

If $D^{*}$ is not connected near $T$, there exists a point $P$ of $T$, and a circle $K_{3}$ about $P$ such that within every circle concentric with $K_{3}$, but having a smaller radius, there exist a pair of points of $D^{*}$ which cannot be joined by a connected subset, lying entirely within $K_{3}$, of $D^{*}$. Since $H$ is connected near $T$, there exists within $K_{3}$ a circle $K_{4}$, concentric with $K_{3}$, such that any two points of $H$ within $K_{4}$ can be joined by a connected subset of $H$, lying entirely within $K_{3}$. There exists an integer $n$ greater than $m+1$ such that $J(n, P)$ is a subset of the interior of $K_{4}$. There exists within $D(n, P)$ a pair, $t_{1}$ and $t_{2}$, of points of $D^{*}$ which cannot be joined by a connected subset of $D^{*}$, lying entirely within $K_{3}$. The points $t_{1}$ and $t_{2}$ are points or boundary points of two domains $d_{1}$ and $d_{2}$, respectively, which are complementary domains of the point set $K_{1}$, and which are therefore subsets of $D(n, P)$. From the definition of $K_{1}{ }^{*}$ it follows that there exist a pair of points $w_{1}$ and $w_{2}$ of $H$, which are points or limit points of $d_{1}$ and $d_{2}$ respectively. There 
exists a connected subset $w_{1} w_{2}$ of $H$, containing $w_{1}$ and $w_{2}$, and lying entirely within $K_{3}$. The point set $t_{1}+d_{1}+w_{1} w_{2}+d_{2}+t_{2}$ is a connected subset of $D^{*}$, and lies entirely within $K_{3}$. Thus, the supposition that $D^{*}$ is not connected near $T$ has led to a contradiction.

By Theorem 6, $K_{2}-T$ is connected. By a theorem of Moore's $\dagger$ there exists a simple closed curve $J$ which separates $H$ from $K_{2}-T$, contains $T$, and, except for $T$, is a subset of $D$.

We have thus established the conclusion for the case where $\bar{H}$ is a continuum and $H$ is connected near $T$. We shall next show that if $H$ is any point set whatever satisfying the hypothesis, then there exists a point set $H^{*}$ having the properties we have so far assumed for $H$. Let $E_{n}, C_{n}, \bar{y}$, and $m$ have the same significance as before, with the exception that the fifth part of the definition of $C_{n}$ and $E_{n}$ read as follows: (5') If $t_{1}$ and $t_{2}$ are points of $H$ in or on the boundary of a maximal domain $D^{*}$ of $E_{n}$, then there exists within that domain of $E_{n-1}$ containing $D^{*}$ a connected subset $t_{1} t_{2}$ of $D$ containing $t_{1}$ and $t_{2}$. By the Heine-Borel-Lebesgue Theorem there exists within $D$ a closed point set, $\bar{A}_{m+2}$, consisting of a finite number of circles plus their interiors, such that all points of $H$ without $E_{m+2}$ belong to $\bar{A}_{m+2}$. There exist at most a finite number of maximal connected subsets of $\bar{A}_{m+2}$. There exists within $D$ a finite collection, $\bar{F}_{m+2}$, of simple continuous arcs, such that $\bar{A}_{m+2}+\bar{F}_{m+2}$ is connected. There exists in $D$ a finite collection, $\bar{G}_{m+2}$, of arcs, such that $\bar{A}_{m+2}+\bar{F}_{m+2}+\bar{G}_{m+2}$ is connected, and that every maximal domain of $E_{m+2}$ contains a point common to $H$ and to $\bar{G}_{m+2}$. There exists a sequence $n_{1}(=m$ $+2), n_{2}, n_{3}, \cdots$ of positive integers, such that for each positive integer $i$, $n_{i}+2<n_{i+1}$, and such that if $d_{i}$ is a maximal domain of the collection $E_{n_{i}}$, then $d_{i}$ contains a point of $H$ which does not belong to any domain of the collection $E_{n_{i+1}}$. Let $M_{n_{i}}$ be the set of all points which belong to some domain of the collection $E_{n i}$ or the boundary of such a domain, but belong to no domain of the collection $E_{n_{i+2}}$. The set of points common to $M_{n_{i}}$ and $H$ is closed and can be covered by a finite set of Jordan regions, each region containing a point of $H \cdot M_{n_{i}}$ such that if $A_{n_{i}}$ denotes the sum of these regions plus their boundaries, then $A_{n i}$ is a subset of both $D$ and $M_{n i-1}+M_{n_{i}}+M_{n_{i+1}}$. There exists by part (5) of the definition of $E_{n}$ and Theorems 10 and 15, F. A., a finite collection of simple continuous $\operatorname{arcs,}$, whose $\operatorname{sum} F_{n_{i}}$ is a subset of $D$, and contains no point without $E_{n_{i-1}}$, such that if $d_{i}$ is a maximal domain of $E_{n_{i}}$ and $R_{1}$ and $R_{2}$ are connected subsets of $A_{n_{i}}$ containing points in common with $d_{i}$, then there exists a closed and connected subset of $A_{n_{i}}+F_{n_{i}}$

$\dagger$ See Theorem 2, Concerning the separation of point sets by curves, loc. cit. 
containing $R_{1}$ and $R_{2}$, and lying entirely within that maximal domain of $E_{n_{i}-1}$ which contains $d_{i}$. Let

$$
H^{*}=\bar{A}_{m+2}+\bar{F}_{m+2}+\bar{G}_{m+2}+\sum_{i=1}^{\infty}\left(A_{n i}+F_{n_{i}}\right) .
$$

Then $H^{*}$ contains $B$, is a subset of $D$, is connected, and is connected near $T$; also, $\bar{H}^{*}=H^{*}+T$. Hence, the condition in our theorem is sufficient. It follows from Theorem 15 that the condition is also necessary.

THEOREM 17. If $K$ and $H$ are bounded continua which have in common $a$ totally disconnected point set $T$, then a necessary and sufficient condition that there exist a simple closed curve which separates $K-T$ from $B-T$ is that $B-T$ is not separated by $K$ near $T$.

By a method of argument analogous to that used in proving Theorem 8 it can be shown that $H-T$ is a subset of a complementary domain of $D$. The truth of the theorem follows from Theorem 16.

THEOREM 18. If $T$ is a closed, totally disconnected subset of a bounded continuum $K$, and $K-T=K_{1}+K_{2}$, where $K_{1}$ and $K_{2}$ are mutually separated point sets, $x$ is a point of $K_{1}$ and $y$ of $K_{2}$, then there exists a simple closed curve which separates $x$ from $y$ and contains in common with $K$ only points of $T$ which are limit points of $K_{1}$.

Let $T_{1}=T \cdot \bar{K}_{1}$. Then $K-T_{1}=K_{1}+K_{2}+T-T_{1}$. It follows that $T_{1}$ is closed and totally disconnected and that $K_{1}$ and $K_{2}+T-T_{1}$ are mutually separated point sets. Let $F y$ be a simple continuous arc containing no point of $K_{1}$, and having end points $y$ and $F$, where $F$ is a point of $S-K$. Let $C$ be a circle with center at $F$, whose interior contains no point of $K, I$ be an inversion of the plane with respect to the circle $C$, and $H^{*}, K^{*}, T^{*}, r^{*}, x^{*}$, and $y^{*}$ be the transforms under this inversion of $\overline{K_{2}+T-T_{1}}, \bar{K}_{1}, T_{1}, F y-F$, $x$, and $y$. By methods similar to those used in the proof of Theorem 2 of Moore's paper Concerning the separation of point sets by curves, $\dagger$ we can show the existence of a simple closed curve $J^{*}$ which separates $x^{*}$ from $y^{*}$, encloses $x^{*}$, and contains no points of $H^{*}+K^{*}-T^{*}$. It is to be noted that both our hypothesis and our conclusion are weaker than Moore's, and that we are not concerned with the latter part of his argument. The inverse under $I$ of $J^{*}$ will be a simple closed curve of the type specified in the conclusion of the theorem.

Theorems 19-24, following, are corollaries of Theorem 18.

$\dagger$ Loc. cit., pp. 470-471. 
THEOREM 19. The conclusion of the preceding theorem remains true if the condition that $K$ be bounded is removed, and the curve $J$ be allowed to be either a simple closed curve or an open curve.

THEOREM 20. If $K$ is a closed point set which consists of a collection of unbounded continua, and $K=K_{1}+K_{2}$, where $K_{1}$ and $K_{2}$ are mutually separated, and $x$ is a point of $K_{1}$ and $y$ is a point of $K_{2}$, then there exists an open curve which separates $x$ from $y$ and contains no point of $K$.

Theorems 19 and 20 may be proved by performing an inversion of the plane about some sufficiently small circle with center at a point $P$ which does not belong to $K$, and applying Theorem 18 .

Theorem 21. If $K$ is a bounded continuum and $P$ is a cut point $\dagger$ of $K$, then there exists a simple closed curve which separates $K-P$; if $K$ is an unbounded continuum there exists either a simple closed curve or an open curve which separates $K-P$.

THEOREM 22. A cut point of a continuum is accessible from some complementary domain of that continuum.

THEOREM 23. If $D$ is a simply connected domain, $B$ is the boundary of $D$, and $P$ is a cut point of $B$, then there exists either (1) a simple closed curve $J$ which separates $B-P$, and which, except for $P$, is a subset of $D$, or (2) an open curve $C$ which separates $B-P$ such that one ray on $C$ from $P$ is, except for $P, a$ subset of $D$. If $B$ is bounded, then condition (1) of the conclusion holds.

THEOREM 24. A cut point of the boundary of a simply connected domain is accessible from that domain.

$\dagger$ The point $P$ is a cut point of a connected point set $K$, if $K-P$ is not connected.

$\ddagger$ Cf. Theorem 12.

UNIVRSity OF TEXas,

Austin, Texas 